Draft VERSION July 16, 2018

Preprint typeset using $\mathrm{IAT}_{\mathrm{E}} \mathrm{X}$ style emulateapj v. 5/2/11

\title{
RECOVERY OF THE CANDIDATE PROTOPLANET HD 100546 b WITH GEMINI/NICI AND DETECTION OF ADDITIONAL (PLANET-INDUCED?) DISK STRUCTURE AT SMALL SEPARATIONS
}

Thayne Currie ${ }^{1}$, Takayuki Muto ${ }^{2}$, Tomoyuki Kudo ${ }^{1}$, Mitsuhiko Honda $^{3}$, Timothy D. Brandt ${ }^{4}$, Carol Grady ${ }^{5}$, Misato Fukagawa ${ }^{6}$, Adam Burrows ${ }^{7}$, Markus Janson ${ }^{8}$, Masayuki Kuzuhara ${ }^{9}$, Michael W. McElwain ${ }^{10}$, Katherine Follette ${ }^{11}$, Jun Hashimoto ${ }^{12}$, Thomas Henning ${ }^{13}$, RYo KAndori ${ }^{14}$, Nobuhiko Kusakabe ${ }^{14}$, Jungmi KWON $^{15}$, Kyle Mede ${ }^{15}$, Jun-IChi Morino ${ }^{14}$, Jun Nishikawa ${ }^{14}$, Tae-Soo Pyo ${ }^{1}$, Gene Serabyn ${ }^{16}$, TakuYa Suenaga ${ }^{14}$, Yasuhiro Takahashi ${ }^{14,15}$, John Wisniewski ${ }^{12}$, Motohide Tamura ${ }^{14,15}$

Draft version July 16, 2018

\section{ABSTRACT}

We report the first independent, second-epoch (re-)detection of a directly-imaged protoplanet candidate. Using $L^{\prime}$ high-contrast imaging of HD 100546 taken with the Near-Infrared Coronagraph and Imager (NICI) on Gemini South, we recover 'HD 100546 b' with a position and brightness consistent with the original VLT/NaCo detection from Quanz et al, although data obtained after 2013 will be required to decisively demonstrate common proper motion. HD 100546 b may be spatially resolved, up to $\approx 12-13 \mathrm{AU}$ in diameter, and is embedded in a finger of thermal IR bright, polarized emission extending inwards to at least $0{ }^{\prime \prime} 3$. Standard hot-start models imply a mass of $\approx 15 M_{J}$. But if HD $100546 \mathrm{~b}$ is newly formed or made visible by a circumplanetary disk, both of which are plausible, its mass is significantly lower (e.g. 1-7 $M_{J}$ ). Additionally, we discover a thermal IR-bright disk feature, possibly a spiral density wave, at roughly the same angular separation as HD $100546 \mathrm{~b}$ but 90 degrees away. Our interpretation of this feature as a spiral arm is not decisive, but modeling analyses using spiral density wave theory implies a wave launching point exterior to $\approx 0$ ". 45 embedded within the visible disk structure: plausibly evidence for a second, hitherto unseen wide-separation planet. With one confirmed protoplanet candidate and evidence for 1-2 others, HD 100546 is an important evolutionary precursor to intermediate-mass stars with multiple super-jovian planets at moderate/wide separations like HR 8799.

Subject headings: planetary systems, stars: early-type, stars: individual: HD 100546

\section{INTRODUCTION}

Nearly all stars are born surrounded by gas and dust-rich protoplanetary disks comprising the building blocks for young gas giant planets (e.g. Hernández et al. 2007; Cloutier et al. 2014). Over the past 7 years, di-

${ }^{1}$ NAOJ, Subaru Telescope, 650 N' Aohoku Pl., Hilo, HI 96720, currie@naoj.org

${ }^{2}$ Kogashin University

3 Kanagawa University

${ }^{4}$ Astrophysics Department, Institute for Advanced Study, Princeton, NJ, USA

${ }^{5}$ Eureka Scientific, 2452 Delmer, Suite 100, Oakland CA96002, USA

${ }^{6}$ Graduate School of Science, Osaka University, 1-1 Machikaneyama, Toyonaka, Osaka 560-0043, Japan

${ }^{7}$ Department of Astrophysical Sciences, Princeton University, 7 Ivy Lane, Princeton, NJ

8 Stockholm University

${ }^{9}$ Department of Earth and Planetary Sciences, Tokyo Institute of Technology, 2-12-1 Ookayama, Meguro-ku, Tokyo 1528551, Japan

10 Exoplanets and Stellar Astrophysics Laboratory, Code 667, Goddard Space Flight Center, Greenbelt, MD 20771, USA

${ }^{11}$ Department of Astronomy, Steward Observatory, University of Arizona

${ }^{12}$ H. L. Dodge Department of Physics \& Astronomy, University of Oklahoma, 440 W Brooks St Norman, OK 73019, USA

13 Max Planck Institute for Astronomy, Konigstuhl 17, 69117 Heidelberg, Germany

${ }^{14}$ National Astronomical Observatory of Japan, 2-21-1, Osawa, Mitaka, Tokyo, 181-8588, Japan

${ }^{15}$ Department of Astronomy, The University of Tokyo, 7-3-1, Hongo, Bunkyo-ku, Tokyo, 113-0033, Japan

16 Jet Propulsion Laboratory, 4800 Oak Grove Drive, MS 183900, Pasadena, CA 91109, USA rect imaging observations have revealed a dozen fullyformed (candidate) super-jovian planets $\left(\begin{array}{lll}5-15 & M_{J}\end{array}\right)$ around young stars (e.g. Marois et al. 2008, 2010a; Lagrange et al. 2010; Carson et al.|2013; Kuzuhara et al. 2013; Rameau et al. 2013; Currie et al. 2014a). Direct images of protoplanets embedded in disks provide a critical link between planet formation's starting point and these various giant planet formation outcomes.

Numerous protoplanetary disks show strong but indirect evidence for infant jovians (e.g. Muto et al. 2012; Grady et al. 2013; Quanz et al. 2013a; Garufi et al. 2013). However, no protoplanets have yet been independently confirmed in multiple epochs. Some candidates e.g. T Cha b, LkCa 15 b-are identified from nonzero closure phase signals from sparse aperture masking observations (Huélamo et al. 2011; Kraus and Ireland 2012), but have yet to be confirmed by other authors or can be misidentified disk signals (Olofsson et al. 2013; Cieza et al. 2013). Two data sets (Reggiani et al. 2014; Biller et al. 2014) identified the candidate HD $169142 \mathrm{~b}$ at $r \approx 1-2 \lambda / \mathrm{D}$ (but at conflicting positions) with the same setup (camera, $t_{\text {int }}$, processing) and at the same epoch. While promising, this candidate is therefore susceptible to the same misinterpretations (e.g. a PSF artifact; a partially-subtracted disk; $\mathrm{PAH}$ emission, see Biller et al. 2014).

In contrast, key features of the protoplanet candidate around HD 100546 (HD 100546 b; Quanz et al. 2013b), make it more amenable to follow up and confirmation 17 .

17 Note that exoplanets.eu equates "HD 100546 b" with the 
HD 100546 was directly imaged in a single epoch (30 May $2011)$ at $\sim 5 \lambda / \mathrm{D}\left(r \sim 0\right.$ !' 48 or $\left.r_{\text {proj }} \sim 47 \mathrm{AU}\right)$, not $\sim 1-2$ $\lambda / \mathrm{D}$, just exterior to a region of polarized disk emission (Avenhaus et al. 2014). High-resolution near-IR CO and $\mathrm{OH}$ spectroscopy provides evidence for another, hitherto unseen, companion at $r \approx 10$ AU (Brittain et al. 2014). ALMA data show evidence for two rings of mm-sized dust, possibly trapped by these candidate protoplanets (Walsh et al. 2014). At regions exterior to the likely jovian planet formation region $(r>100 \mathrm{AU})$, the disk may also exhibit planet-induced structure (Grady et al. 2005; Boccaletti et al. 2013).

New observations with a different telescope/set-up can 1) confirm HD 100546 b's existence as an embedded point source-like object and 2) identify additional signatures of protoplanets from thermal IR bright disk features at similar angular separations. Like several stars with imaged planets (HR 8799, $\beta$ Pic, and HD 95086), HD 100546 is an intermediate-mass star and thus provides a critical look at the formation of super-jovian planets at moderate/wide separations.

In this Letter, we report the recovery/confirmation of embedded protoplanet candidate HD $100546 \mathrm{~b}$ from Gemini/NICI $L^{\prime}$ data at the same location and brightness as reported in Quanz et al. (2013b). Additionally, we identify a new thermal IR-bright disk feature at roughly the same angular separation, possibly linked to another actively-forming planet.

\section{OBSERVATIONS AND DATA REDUCTION}

We observed HD 100546 on 31 March 2012 with the Near-Infrared Coronagraph and Imager (NICI) on Gemini-South (Chun et al. 2008) in the $K_{s},[3.1]$ and $L^{\prime}$ filters (0.'0179/pixel). A future study includes contemporaneous $K_{s}$ and [3.1] imaging of HD 100546 and presents a comprehensive analysis of the disk (Honda et al. in prep.). Our data consist of coadded 19-second exposures taken in a three-point dither pattern in angular differential imaging mode (Marois et al. 2006) (hour angle $=[-3.6,-0.86]$ ), yielding $49.5^{\circ}$ of parallactic motion. The stellar PSF was stable and sky transmission was typically constant to within $5-10 \%$. We identified 2128 $\mathrm{s}$ of good-quality science data. Basic image processing steps followed our previous methods for reducing thermal IR data (Currie et al. 2011, 2014b). For each image, we subtracted a moving-box median filter of width $5 \lambda / \mathrm{D}$ to remove low spatial frequency noise and axisymmetric disk halo emission.

We performed PSF subtraction using the A-LOCI pipeline (Currie et al. 2014b), subtracting each science frame by a linear combination of reference images obtained at different parallactic angles $(\Delta \mathrm{PA} \geq$ $\delta \times \mathrm{FWHM}$ ), where the images' coefficients are determined by solving the set of linear equations $\mathbf{x}=\mathbf{A}^{-1} \mathbf{b}$. For a second, independent approach we reduced the data using the Karhoeven-Loéve Image Projection (KLIP) algorithm (Soummer et al. 2012). We construct eigenimages in annular regions, retaining $n_{\text {pca }}$ principal components, where $n_{\text {pca }}<n_{\text {images }}$. As with A-LOCI, we impose a rotation gap criterion $(\delta)$.

proposed companion at $10 \mathrm{AU}$ (Brittain et al.|2014). Throughout, we follow the naming conventions from Quanz et al. (2013b).

\section{DETECTIONS}

Figure 1 displays our images reduced using A-LOCI (left) and KLIP (right), showing a point source-like peak at the position of HD $100546 \mathrm{~b}$ (Quanz et al. 2013b) superimposed upon an extended finger of disk emission previously seen in total and polarized intensity (Quanz et al. 2013b; Avenhaus et al. 2014). Both reductions also reveal a bright, spiral arm-like disk structure 90 degrees away, starting at $r \sim 0.33, P A \approx 150^{\circ}$ near the disk semi-major axis (Avenhaus et al. 2014) to $r \sim 00^{\prime \prime} 6, P A$ $\approx 90^{\circ}$. Furthermore, the KLIP reduction (and A-LOCI reduction to a lesser extent) shows what appears to be a brightness peak superimposed on the structure at 0.4 0 !' 4518. Negative subtraction footprints flank the positions of HD $100546 \mathrm{~b}$ and the disk feature, consistent with them being real astrophysical features attenuated by image processing (e.g. Marois et al. 2010b; Milli et al. 2012; Brandt et al. 2013).

To compute signal-to-noise (SNR), we use standard methods, replacing each pixel with the signal integrated over an aperture equal to that of a point source (FWHM $\sim 0^{\prime \prime} .1$ ) and comparing this to the integrated signal at the same separation but other position angles (e.g. Thalmann et al. 2009; Currie et al. 2014b). Our A-LOCI and KLIP reductions recover HD $100546 \mathrm{~b}$ at $\mathrm{SNR}=5.3$ (Figure 2, left panel) and 4.0, respectively.

In both reductions, the disk feature appears as a clear SNR $\gtrsim 3$ trail beyond $\sim 0^{\prime \prime} .3$. We also detected it in archival $2011 \mathrm{NaCo} L^{\prime}$ data (Figure 1) bottom-right panel) published by Quanz et al. (2013b). Thus, the feature is not a PSF artifact 19 .

Our SNR ratios are conservative and our detection of HD 100546 is probably comparable to the original VLT/NaCo detection from Quanz et al. (2013b) 20. We do not mask the negative subtraction footprints, nor do we mask the candidate spiral when determining the SNR for HD $100546 \mathrm{~b}$ and vice versa. The radial profile of the residual noise is flat at $r \sim 0.0^{\prime \prime} 4-0.5$ but drops steeply at $r$ $>0.5$ (overlapping with the outer half of HD 100546 b's PSF). Fixing the centroid position to be one pixel (two pixels) further from the star increases the SNR in the A-LOCI and KLIP reductions to 6.2 (7) and 5.0 (5.6), respectively.

Finally, following Currie et al. (2014b), we computed the probability distribution function (PDF) for elements on our signal-to-noise map within a $1 \lambda / \mathrm{D}$-wide ring enclosing HD 100546 b (Figure 2, right panel). The inten-

18 Avenhaus et al. (2014) identify a candidate spiral at $r \sim 0^{\prime \prime} .2-$ $0 . \prime 3$. Our A-LOCI reduction reveals a similar feature appearing to curve in the direction of HD $100546 \mathrm{~b}$, its signal is not statistically significant. Thus, we do not consider it in this paper.

19 We also easily recover HD $100546 \mathrm{~b}$ and the disk feature in unpublished $2013 L^{\prime} \mathrm{NaCo}$ data (PI. S. Quanz). Other authors will present these results.

20 Quanz et al. (2013b) report a SNR $\sim 15$ detection using a different definition for SNR, $S / N \sim S_{\text {integrated }} /\left(\sigma_{\text {pixel }} \times\right.$ $\left.\sqrt{\pi \times r_{\text {aperture }}^{2}}\right)$, which is valid for noise uncorrelated at the pixelto-pixel level (e.g. photon noise). This condition is generally not satisfied in high-contrast imaging data, where both quasi-static speckles in raw images and the power spectrum of residual speckle noise in processed images peaks at scales much larger than one pixel (Figure 9 in Brandt et al.|2013): the relevant size scale is the resolution element, $\lambda / \mathrm{D}$ (Mawet et al. 2014). Using Quanz et al.'s formalism, our SNR increases to SNR $\sim 15.2$ but yields 9 other $5+\sigma$ peaks within $0^{\prime \prime} 75$. 
sity residuals are almost perfectly normally distributed. While the finite number of resolution elements at HD 100546 b's separation $(\sim 30)$ results in a somewhat higher false alarm probability than would be inferred from a perfect gaussian distribution (Mawet et al.|2014), masking real features (e.g. the disk) would again narrow the distribution, increase the SNR, and slightly lower the false alarm probability. Using Eq. 9 in Mawet et al. (2014), the SNR exceeds 5- $\sigma$.

\section{ANALYSIS}

\subsection{Properties of HD $100546 \mathrm{~b}$}

\subsubsection{Size of Emitting Area and Position}

We computed HD 100546 b's apparent FWHM from moving-box median-filtered versions of both reductions (to remove residual disk emission) following Kenyon et al. (2014). HD 100546 b is slightly extended compared to the predicted size of a point source $(\sim$ 0 "' 1 or 5.6 pixels $): \mathrm{FWHM}_{x, y}=6.8,7.6$ pixels $(0$ ' 12 , 0 .'14) for the A-LOCI reduction and $\mathrm{FWHM}_{x, y}=7.2$, $7.6\left(00^{\prime \prime} 13,0.14\right)$ pixels for the KLIP reduction, implying a diameter of up to $12-13 \mathrm{AU}$. Both processing methods can slightly attenuate emission in the azimuthal direction (e.g. Marois et al. 2010b). A lack of a good PSF model (see below) precludes our ability to better estimate HD 100546 b's size and its debias astrometry. However, using synthetic point sources with comparable apparent sizes, we verified that attenuation is extremely weak at HD 100546 b's separation (throughput $\approx 70,85 \%$ for A-LOCI and KLIP).

HD 100546 b's position is $[\mathrm{E}, \mathrm{N}]^{\prime \prime}=[0.083,0.477]^{\prime \prime}$ \pm 0 .'012 for the A-LOCI reduction and $[0.084,0.468]^{\prime \prime}$ $\pm 0^{\prime \prime}$.013 for the KLIP reduction $\left(r_{\text {proj }} \sim 47\right.$ AU, $\left.\theta \sim 10^{\circ}\right)$ : nearly identical to those reported by Quanz et al. (2013b). The small time difference between Quanz et al.'s discovery observations and our data cannot prove common proper motion. However, HD 100546 b's position within the disk makes the companion extremely unlikely to be a background object.

\subsubsection{Brightness and Estimated Mass}

We can obtain only rough estimates for HD 100546 b's brightness. Our photometric calibrator BS4638 $\left(\mathrm{m}_{L^{\prime}}=\right.$ 4.5) was observed with the AO loop off, HD 100546A's PSF core was saturated, and we were unable to obtain other unsaturated NICI PSF images. For a model HD 100546 b PSF, we simply constructed a gaussian with $\mathrm{FWHM}_{x, y}=[7,7.6]$ pixels and compared the encircled energy between a 3-pixel radius aperture and an infinite one to estimate an aperture correction. We then injected negative copies of the model PSF at the HD 100546 b's position and identified the range of magnitudes where the signal is clearly oversubtracted/undersubtracted, defining the estimated magnitude as the range's midpoint and equating the intrinsic photometric uncertainty with this range. Photometric errors consider HD 100546 b's SNR, the photometric calibrator's SNR, and uncertainties in the throughput correction. We derive $\mathrm{m}_{L^{\prime}, b}=13.06 \pm$ 0.51 , consistent with Quanz et al. (2013b).

HD 100546 b plausibly has a mass well below the deuterium-burning limit. Given an age of 5-10 $\mathrm{Myr}$, a distance of $97 p c$ (van Leeuwen 2007), and using standard hot-start models to estimate planet mass
(Spiegel and Burrows 2012), our photometry and the Boccaletti et al. upper limits imply a mass of $\approx 15 \mathrm{MJ}_{J}$. However, HD 100546 b could be newly born, significantly younger than the star (see Currie et al. 2013). For $t=$ 1 Myr, the Spiegel and Burrows models nominally yield $M_{\mathrm{b}} \sim 7 M_{J},<10 M_{J}$ for an initial entropy of $S_{\text {init }}>$ $11.75 k_{B}$ /baryon.

If HD $100546 \mathrm{~b}$ is resolved, much (all?) of its emission could come from an accreting circumplanetary disk (e.g. Fujii et al. 2014; Zhu 2014) and its mass could be significantly below $10 M_{J}$. Assuming a mass of $1 M_{J}$, a truncated disk with $R_{\text {in }}=1.5-2 R_{J}$, and a circumplanetary accretion rate to $5-10 \%$ that of the circumstellar disk (or $\dot{M}_{b} \approx 3-6 \times 10^{-6} M_{\mathrm{J}} y r^{-1}$, c.f. Pogodin et al. 2012), the disk can reproduce our $L^{\prime}$ photometry without making HD $100546 \mathrm{~b}$ detectable at $K_{s}$.

\subsection{Spiral Arm Fitting of the Newly-Discovered Thermal IR-Bright Disk Structure: Evidence for a Second Wide-Separation Planet?}

Although the morphology of the newly-identified disk feature at $r>0$ ". 35 lacks conclusive results, it resembles spiral arms in HD 100546's disk at other separations (Grady et al. 2005; Ardila et al. 2007; Avenhaus et al. 2014) and in other protoplanetary disks (Muto et al. 2012; Grady et al. 2013). Unlike these spirals, this feature appears to be particularly bright in the thermal IR but less easily identifiable in polarized light (c.f. Avenhaus et al. 2014). Thus, any spiral features primarily trace the disk structure in total intensity, not polarized intensity.

We model this feature using spiral density wave theory following the methodology outlined in Muto et al. (2012), fitting the spiral shape, which depends on the wave launching point $\left(r_{o}, \theta_{o}\right)$; the disk aspect ratio $\left(h_{c}\right)$ at the launching point; the radial power-law index for the rotation angular velocity, $\alpha$; and the radial powerlaw index for the sound speed, $\beta$. We set $\alpha=-1.5$ (the Keplerian value) and perform a four-parameter fit varying $r_{o}, \theta_{o}, \mathrm{~h}_{c}$, and 21 .

Although the disk morphologies in the A-LOCI and KLIP reductions are similar, we focus on modeling the KLIP reduction (see discussion in Soummer et al. 2012) and use the A-LOCI simply as a comparison. We adopt a major axis's position angle and inclination of $138^{\circ}$ and $50^{\circ}$, respectively (Avenhaus et al. 2014). The range of "good-fitting solutions" result from a $\Delta \chi^{2}$ criterion and correspond to the $5-\sigma$ confidence level if the errors associated with representative spiral shape positions are independent.

Table 1 summarizes our results, while Figure 3 compares our best-fit parameters to the de-projected KLIPreduced image. Significant model fitting degeneracies allow only modest constraints on most spiral properties. However, the best-fit wave launching point (and the possible location of a perturbing planet) is at $r_{o}, \theta_{o}=0^{\prime \prime} 65$, $93^{\circ}$, within the wave itself. The $5 \sigma$ contours imply that HD $100546 \mathrm{~b}$ is not launching this spiral. Instead, much of the spiral arm region exterior to 0 ". 45 could be the site

21 Our modeling results derive just from the peak signal along the arm. Azimuthal self-subtraction does, however, prevent us from precisely measuring the spiral amplitude, which is connected to planet mass if the spiral is launched by a planet. 
of the wave launching point, including the local maximum most clearly seen in the KLIP reduction. Our results from modeling the A-LOCI reduced image likewise imply a wave launching point exterior to 0 '. 45 , plausibly within the wave but not consistent with HD 100546 b's position. Rerunning our fits using a sparse sampling where the positional errors should be (more) independent yields nearly identical results. If the newly-identified disk feature is a spiral arm induced by a hitherto unseen planet, then the planet is most likely an additional companion exterior to $r \approx 0$ ". 45 .

\section{SUMMARY AND DISCUSSION}

Our study reports the first independent, second-epoch (re-)detection of a directly-imaged protoplanet. Using Gemini/NICI thermal IR data, we recover HD 100546 b at the same location and brightness as in the discovery image (Quanz et al. 2013b). Furthermore, we discover an extended disk feature $90^{\circ}$ from HD $100546 \mathrm{~b}$, resembling a spiral density wave, with a possible thermal IR peak at 0 '! $4-0$ "' 45 . While interpreting this feature is not straightforward, modeling it as a spiral density wave implies a wave launching point, the possible location of another protoplanet, exterior to 0 "' 45 and possibly within the visible wave itself.

Although HD 100546 b is now recovered, its status as a directly-imaged planet is on less firm ground than companions like HR 8799 bcde, since it lacks common proper motion confirmation, may be spatially extended, and could in principle be a partially-subtracted piece of the disk. However, it is too far from the star to be reprocessed disk emission. Its non-detection in polarized light and in near-IR total intensity disfavors explaining it as light scattered by small dust grains: if it is indeed a partially-subtracted disk feature, it is a feature with different dust properties. PAH emission in HD 100546's disk appears to originate at the inner disk rim (13 AU), not out at $\mathrm{r}_{\mathrm{proj}} \sim 47 \mathrm{AU}$ (Geers et al. 2007); thermal instabilities likewise seem implausible (c.f. Biller et al. 2014).

Furthermore, HD $100546 \mathrm{~b}$ and the polarized disk material on which it sits together cannot identify another spiral arm, since it would wind in the opposite direction from bona fide arms at wide separations (Grady et al. 2005) and our candidate arm. After ruling out other alternatives, we conclude HD 100546 b identifies locally- produced thermal emission from either a young protoplanet or dissipation driven from some other, unknown mechanism. HD $100546 \mathrm{~b}$ as a protoplanet naturally explains the ring of mm-sized dust located exterior to it (Walsh et al. 2014).

HD 100546 b's nature may soon be decisively determined. Astrometry from 2013-2014 may demonstrate that HD 100546 b is not a background object. New, multi-wavelength photometry (e.g. $2-5 \mu \mathrm{m}$ ) can clarify whether HD $100546 \mathrm{~b}$ has planet-like colors. Integral field spectroscopy with the Gemini Planet Imager or SPHERE (Macintosh et al. 2008; Beuzit et al. 2008) can show whether HD 100546 b has a spectrum similar to that of the youngest planetary objects (e.g. Bonnefor et al. 2014; Currie et al. 2014a).

The newly-detected, spatially extended inner disk feature/candidate spiral arm requires new data to interpret cleanly. Furthermore, while spiral density waves in disks are often associated with planets, a spiral could have nonplanet origins (Lyra et al. 2014, A\&A submitted). New IR observations may allow us to assess whether a planetdriven spiral is reasonable. Further analysis of other regions of the HD 100546 disk from IR data (Honda et al. in prep.) and the (sub-)millimeter with ALMA (e.g. Walsh et al. 2014) will better place our newly-discovered structure within the context of HD 100546's disk morphology in general, provide clues needed to better interpret HD $100546 \mathrm{~b}$ and the disk structure we first identify.

Our study further cements HD 100546 as a superb laboratory for studying the formation of multiple (super)jovian planets (e.g Quillen 2006). The system contains one bona fide protoplanet candidate at $r_{\text {proj }}=47 \mathrm{AU}$, strong evidence for a candidate at $10 \mathrm{AU}$ (Brittain et al. 2014), and potential evidence for additional planets exterior to $r_{\mathrm{proj}} \approx 45 \mathrm{AU}$ (this work). The full range in (projected) separation is qualitatively similar to the projected separations of HR 8799 bcde (Marois et al.|2010a), the only known imaged multi-planet system. Thus, HD 100546 provides important insight into the formation and early evolution of super-jovian planets at moderate/wide separations around intermediate-mass stars.

We thank Wladimir Lyra, Scott Kenyon, Mickael Bonnefoy, Christian Thalmann, Nienke van der Marel, and the anonymous referee for helpful comments. This research makes use of the ESO archive.

\section{REFERENCES}

Ardila, D. R., Golimowski, D. A., Krist, J. E., et al. 2007, ApJ, 665,512

Avenhaus, H., Quanz, S., Meyer, M. R., et al., 2014, ApJ in press, arxiv: 1405.6120

Beuzit, J.-L., Feldt, M., Dohlen, K., et al., 2008, SPIE, 7014, 41

Biller, B., Males, J., Rodigas, T. J., et al., 2014, ApJ, 792, L22

Boccaletti, A., Pantin, E., Lagrange, A.-M., et al. 2013, A\&A, 560, A20

Bonnefoy, M., Chauvin, G., Lagrange, A.-M., et al. 2014, A\&A, 562,127

Brandt, T. D., McElwain, M. W., Turner, E. L., et al. 2013, ApJ, 764,183

Brittain, S., Carr, J., Najita, J., et al., 2014, ApJ, 791, 136

Carson, J., Thalmann, C., Janson, M., et al., 2013, ApJ,

Cieza, L., A., Lacour, S., Schreiber, M. R., 2013, ApJ, 762, L12

Chun, M., Toomey, D., Wahhaj, Z., et al., 2008, SPIE, 7015

Cloutier, R., Currie, T., Rieke, G., et al., 2014, ApJ in press, arXiv:1408.1724
Currie, T., Burrows, A., Itoh, Y., et al., 2011, ApJ, 729, 128

Currie, T., Debes, J. H., Rodigas, T. J., et al., 2012, ApJ, 760, L32

Currie, T., Burrows, A., Madhusudhan, N., et al., 2013, ApJ, 776, 15

Currie, T., Daemgen, S., Debes, J. H., et al., 2014a, ApJ, 780, L30

Currie, T., Burrows, A., Girard, J., et al., 2014b, ApJ in press, arXiv:1409.5134

Fujii, Y., Okuzumi, S., Tanigawa, T., \& Inutsuka, S.-I., 2014, ApJ, 785, 101

Garufi, A., Quanz, S. P., Avenhaus, H., et al. 2013, A\&A, 560, A 105

Geers, V. C., van Dishoeck, E. F., Visser, R., et al., 2007, A\&A, 476,279

Grady, C. A., Woodgate, B. E., Bowers, C. W., et al. 2005, ApJ, 630,958

Grady, C. A., Muto, T., Hashimoto, J., et al. 2013, ApJ, 762, 48 

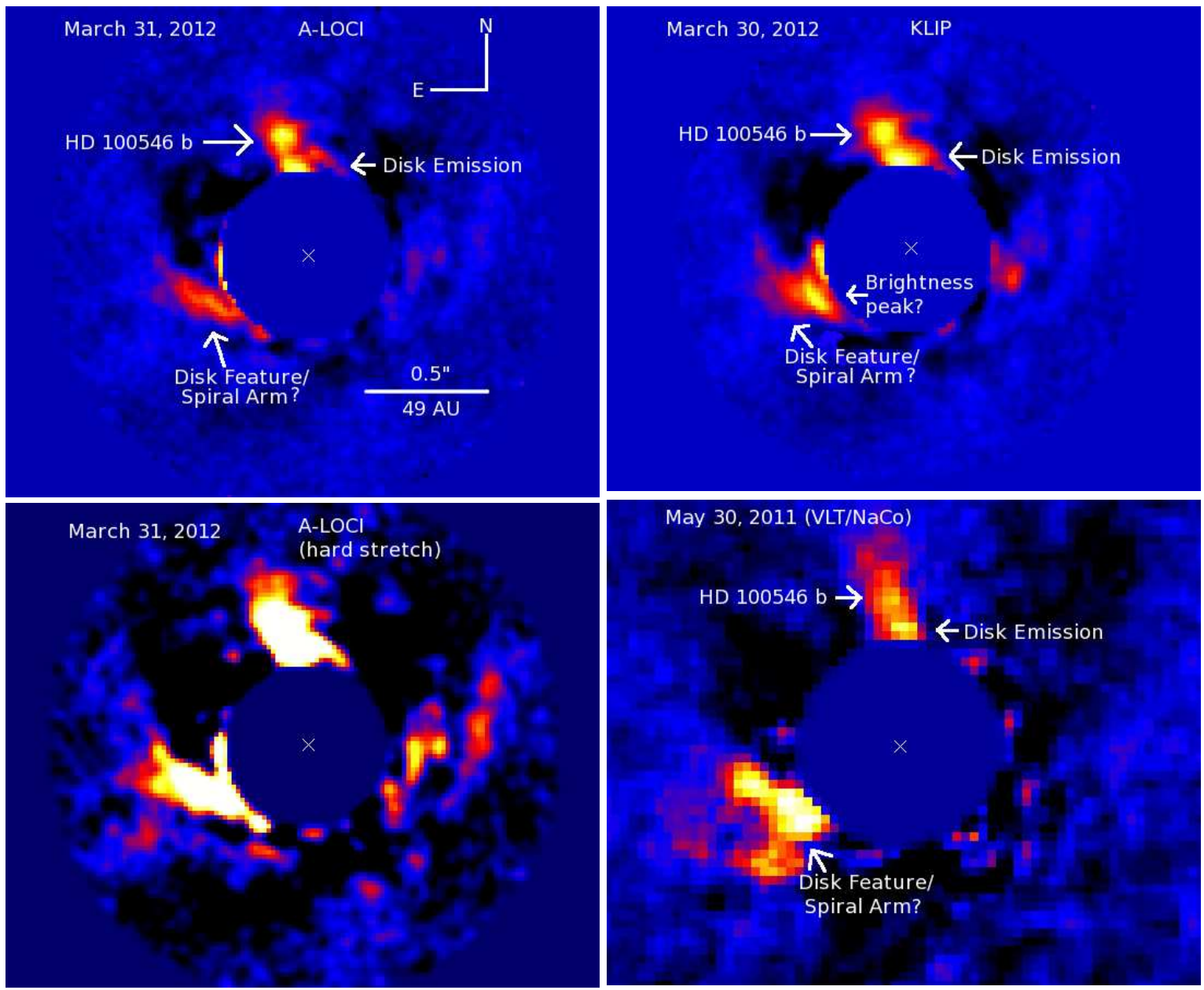

FIg. 1.- Gemini/NICI $L^{\prime}$ image of HD 100546 processed with A-LOCI (top-left) and KLIP (top-right) revealing HD 100546 b and a hitherto undetected disk feature/candidate spiral arm. The A-LOCI reduction adopts a small rotation gap $(\delta=0.55)$ and optimization area $\left(N_{a}=40\right)$ (see Lafrenière et al. 2007) but a large SVD cutoff $\left(7.5 \times 10^{-4}\right)$, aggressive frame selection $\left(n_{\text {ref }}=25\right)$, and a moving pixel mask, resulting in good throughput (see Marois et al. 2010b; Currie et al. 2012, 2014b). The KLIP reduction assumes $\delta=0.9$, where the eigenimages are constructed over 10-pixel wide annuli at a time from the first 5 principal components (see Soummer et al. 2012). (bottom-left) A-LOCI image with a hard color stretch to better show the extent of the disk emission. (bottom-right) We identify this same extended disk features in archival $2011 \mathrm{NaCo} L^{\prime}$ data presented by Quanz et al. .

Hernández, J., Hartmann, L., Megeath, S. T., et al., 2007, ApJ, 662,1067

Huélamo, N., Lacour, S., Tuthill, P., et al. 2011, A\&A, 528, L7

Kenyon, S. J., Currie, T., Bromley, B., 2014, ApJ, 786, 70

Kraus, A. L., \& Ireland, M. J. 2012,ApJ, 745, 5

Kuzuhara, M., Tamura, M., Janson, M., et al., 2013, ApJ, 774, 11

Lafrenière, D., Marois, C., Doyon, R., Nadeau, D.,\& Artigau, É. 2007, ApJ,660, 770

Lagrange, A.-M., et al., 2010, Science, 329, 57

Macintosh, B., A., Graham, J. R., Palmer, D. W., et al. 2008, Proc. SPIE, 7015, 31

Marois, C., Lafrenière, D., Doyon, R., Macintosh, B., \& Nadeau, D. 2006, ApJ, 641, 556

Marois, C., Macintosh, B., Barman, T., et al. 2008, Science, 322, 1348

Marois, C., Zuckerman, B., Konopacky, Q. M., Macintosh, B., \& Barman, T., 2010, Nature, 468, 1080

Marois, C., Macintosh, B.,\& Vèran, J.-P., 2010b, Proc. SPIE, 7736,52

Mawet, D., Wahhaj, Z., Pelat, D., et al., 2014, ApJ, 792, 97

Milli, J., Mouillet, D., Lagrange, A.-M., et al., 2012, A\&A, 545, 111
Muto, T., Grady, C. A., Hashimoto, J., et al., 2012, ApJ, 748, L22 Olofsson, J., Benisty, M., Le Bouquin, J.-B., et al. 2013,A\&A, 552, A4

Pogodin, M. A., Hubring, S., Yudin, R. V., et al., 2012, Astronomische Nachrichten, 333, 594

Rameau, J., Chauvin, G., Lagrange, A.-M., et al., 2013, ApJ, 779, L26

Reggiani, M., Meyer, M., R., Quanz, S., et al., 2014, ApJ, 792, L23

Quanz, S., P., Avenhaus, H., Buenzli, E., et al., 2013a, ApJ, 766, L2

Quanz, S., P., Amara, A., Meyer, M. R., et al., 2013b, ApJ, 766 , L1

Quillen, A., 2006, ApJ, 640, 1078

Soummer, R., Pueyo, L., Larkin, J., 2012, ApJ, 755, L28

Spiegel, D., Burrows, A., 2012, ApJ, 745, 174

Thalmann, C., et al., 2009, ApJ, 707, L123

van Leeuwen, F., 2007, A\&A, 474, 653

Walsh, C., Juhasz, A., Pinilla, P., et al., 2014, ApJ, 791, L6

Zhu, Z., 2014, ApJ in press, arxiv: 1408.6554 

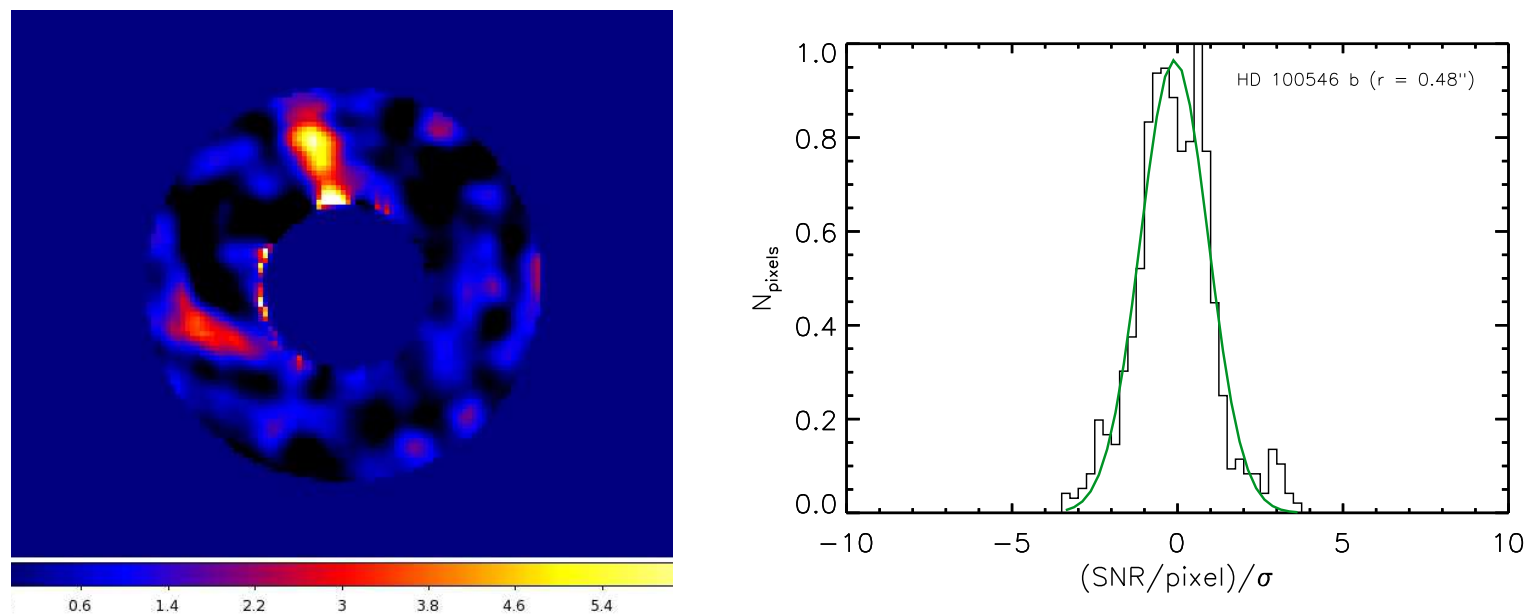

FIG. 2.- (Left) Signal-to-noise map for the A-LOCI reduction: the map derived from our KLIP reduction is similar. HD $100546 \mathrm{~b}$ (SNR $=5.3$ ) sits in an extended finger of emission stretching from $0 . \prime 3$ to at least $0 ! \prime 75$. The map also clearly reveals the disk structure/spiral arm. (Right) Histogram distribution of pixels bracketing the separation of HD $100546 \mathrm{~b}\left(r \sim 00^{\prime \prime} 44-0{ }^{\prime \prime} 54\right)$ with companion's signal masked. The residuals follow a gaussian-like distribution. Most points at 3-4 $\sigma$ trace the spiral arm signal, not residual speckles.
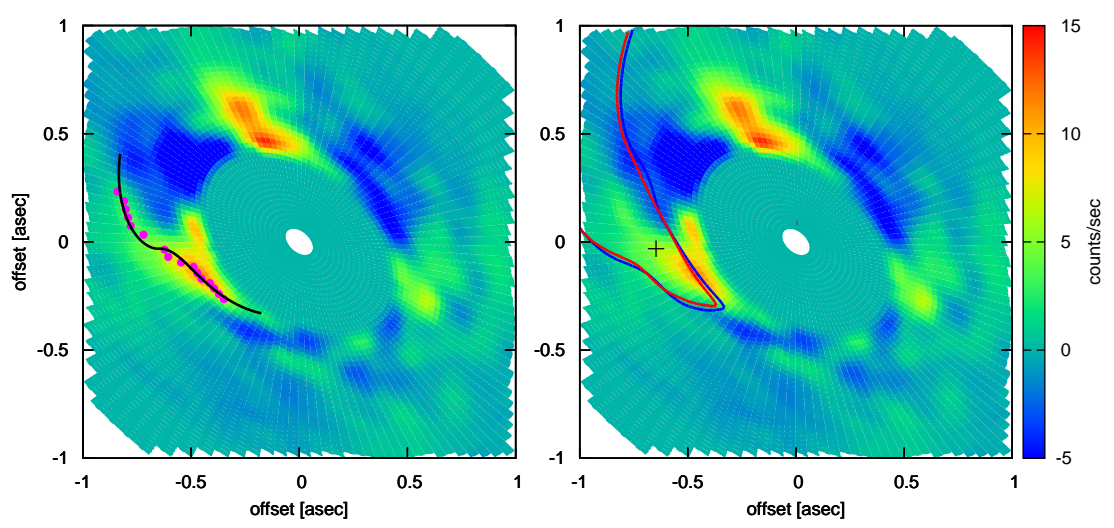

FIG. 3. - Spiral density wave modeling. (Left) The local maxima (magenta dots) tracing the location of the spiral from $r \sim 0$ !' 35 to 0 '. 1 (black line) on the deprojected KLIP image. (Right) The best-fit wave launching point (cross) along with the 5- $\sigma$ contours from the KLIP reduction (blue) and A-LOCI reduction (red).

TABLE 1

Spiral Arm Fitting Results

\begin{tabular}{lllll}
\hline \hline Reduction & $\begin{array}{l}r_{o} \text { (best, range) } \\
\left({ }^{\prime \prime}\right)\end{array}$ & $\begin{array}{l}\theta_{o} \text { (best, range) } \\
\left({ }^{\prime \prime}\right)\end{array}$ & $\begin{array}{l}h_{c} \text { (best, range) } \\
\left({ }^{\circ}\right)\end{array}$ & $\beta$ (best, range) \\
\hline $\begin{array}{l}\text { primary } \\
\text { KLIP }\end{array}$ & $0.65,>0.45$ & $93,<133$ & $0.2,>0.1$ & $0.05,0-1$ \\
$\begin{array}{l}\text { comparison } \\
\text { A-LOCI }\end{array}$ & $0.94,>0.46$ & $76,<133$ & $0.3,>0.1$ & $1,0-1$ \\
\hline
\end{tabular}

Note. - The columns list the best-fit and range for position of the co-rotation radius $\left(r_{o}, \theta_{o}\right)$, the scale height $\left(h_{c}\right)$, and the gas sound speed power law $(\beta)$. 